\title{
Missing baryons, bulk flows, and the E-mode polarization of the Cosmic Microwave Background
}

\author{
C. Hernández-Monteagudo ${ }^{1}$ and R. A. Sunyaev ${ }^{1,2}$ \\ 1 Max Planck Institut für Astrophysik, Karl Schwarzschild Str. 1, 85741 Garching bei München, Germany \\ e-mail: chm@mpa-garching.mpg.de \\ 2 Space Research Institute, Russian Academy of Sciences, Profsoyuznaya 84/32, 117997 Moscow, Russia \\ e-mail: sunyaev@mpa-garching.mpg.de
}

Received 15 May 2008 / Accepted 21 July 2008

\section{ABSTRACT}

\begin{abstract}
Most of the missing baryons are found in slightly overdense structures like filaments and superclusters, but to date most of them have remained hidden to observation. At the same time, the linear cosmological perturbation theory predicts the existence of extended bulk flows seeded by the gravitational attraction of linear potential wells, but again these also remain undetected. If the peculiar motion of galaxy groups and clusters indeed resembles that of the surrounding baryons, then the kinetic Sunyaev-Zel'dovich (kSZ) pattern of those massive halos should be closely correlated to the kSZ pattern of all surrounding electrons. Likewise, it should also be correlated to the CMB E-mode polarization field generated via Thomson scattering after reionization. We explore the cross-correlation of the kSZ generated in groups and clusters to the all sky E-mode polarization in the context of future CMB experiments like Planck, ACT, SPT or APEX. We find that this cross-correlation effectively probes redshifts below $z=3-4$ (where most of the baryons cannot be seen), and that it arises on very large scales $(l<10)$. The significance with which this cross-correlation can be measured depends on the Poissonian uncertainty associated with the number of halos where the kSZ is measured and on the accuracy of the kSZ estimations themselves. Assuming that Planck can provide a cosmic variance limited E-mode polarization map at $l<20$ and $S / N \sim 1 \mathrm{kSZ}$ estimates can be gathered for all clusters more massive than $10^{14} M_{\odot}$, then this cross-correlation should be measured at the 2-3 $\sigma$ level. Further, if an all-sky ACT or SPT type CMB experiment provides similar kSZ measurements for all halos above $10^{13} M_{\odot}$, then the cross-correlation total signal to noise $(S / N)$ ratio should be at the level of 4-5. A detection of this cross-correlation would provide direct and definite evidence of bulk flows and missing baryons simultaneously.
\end{abstract}

Key words. cosmology: cosmic microwave background - cosmology: large-scale structure of Universe

\section{Introduction}

One of the fundamental predictions of the standard cosmological model is that baryons (and matter in general) should be moving in extended bulk flows generated by the gravitational pull of large scale overdense regions. Despite being potentially useful for cosmological studies (Kashlinsky \& Atrio-Barandela 2000; Atrio-Barandela et al. 2004, 2008), none of those bulk flows have been detected to date (Benson et al. 2003), partially due to the difficulty of detecting baryonic matter in the local universe. Indeed, the visible baryonic matter in the various frequency ranges amounts only to one ninth of the total budget of baryons predicted by the cosmological model (Fukugita \& Peebles 2004, this is the so called missing baryon problem). All these baryons must be found in the present Universe, since they have been detected at large $(z \simeq 1100)$ and intermediate redshift $(z \sim 3-6)$, by means of Cosmic Microwave Background (CMB) (Hinshaw et al. 2008) and Lyman- $\alpha$ forest (Croft et al. 1998; McDonald et al. 2005) observations. However, no conclusive observational evidence has been found at low redshift. Some indications have been derived from the observation of absorption lines in the direction of quasars (see Nicastro et al. (2005a,b) and references therein), or a soft X-ray excess around clusters (e.g., Kull \& Böhringer 1999; Zappacosta et al. 2002). Cen \& Ostriker $(1999,2005)$ concluded that most of the baryons are in a warm phase (WHIM), defined by the temperature range
$T \in\left[10^{5}, 10^{7}\right] \mathrm{K}$. Whether most of this gas is in a diffuse phase or in small, unresolved, collapsed objects is still an open question. Recently, Génova-Santos et al. $(2005,2008)$ detected a nonGaussian cold spot when looking at the CMB radiation in the direction of Corona Borealis, and discussed its interpretation in the context of the thermal and the kinetic Sunyaev-Zel'dovich effects. The thermal effect (tSZ, Sunyaev \& Zeldovich 1972) expresses the distortion that the black body CMB spectrum undergoes due to Compton scattering on hot electrons. On the other hand, the kinetic effect (kSZ, Sunyaev \& Zeldovich 1980) describes the Thomson scattering between CMB photons and electrons in which there is no energy exchange (and therefore it introduces thermal or frequency independent brightness temperature fluctuations). In the case of Corona Borealis, it was suggested that a face on filament could give rise, via either tSZ or $\mathrm{kSZ}$, to the pattern seen in the CMB maps. However, it was shown in Hernández-Monteagudo et al. (2006b) that the tSZ is not a useful tool to track the baryons outside the largest collapsed halos: about $\sim 80 \%$ of the tSZ luminosity is generated in the most massive collapsed structures, and the $\sim 50 \%$ of baryons located in underdense or slightly overdense regions give rise to only $\sim 5 \%$ of the total tSZ luminosity. The kSZ is most promising, since it does not require high gas pressure, but relative velocities with respect to the CMB instead (which is itself a prediction of the model). However, the drawback is that the $\mathrm{kSZ}$ 

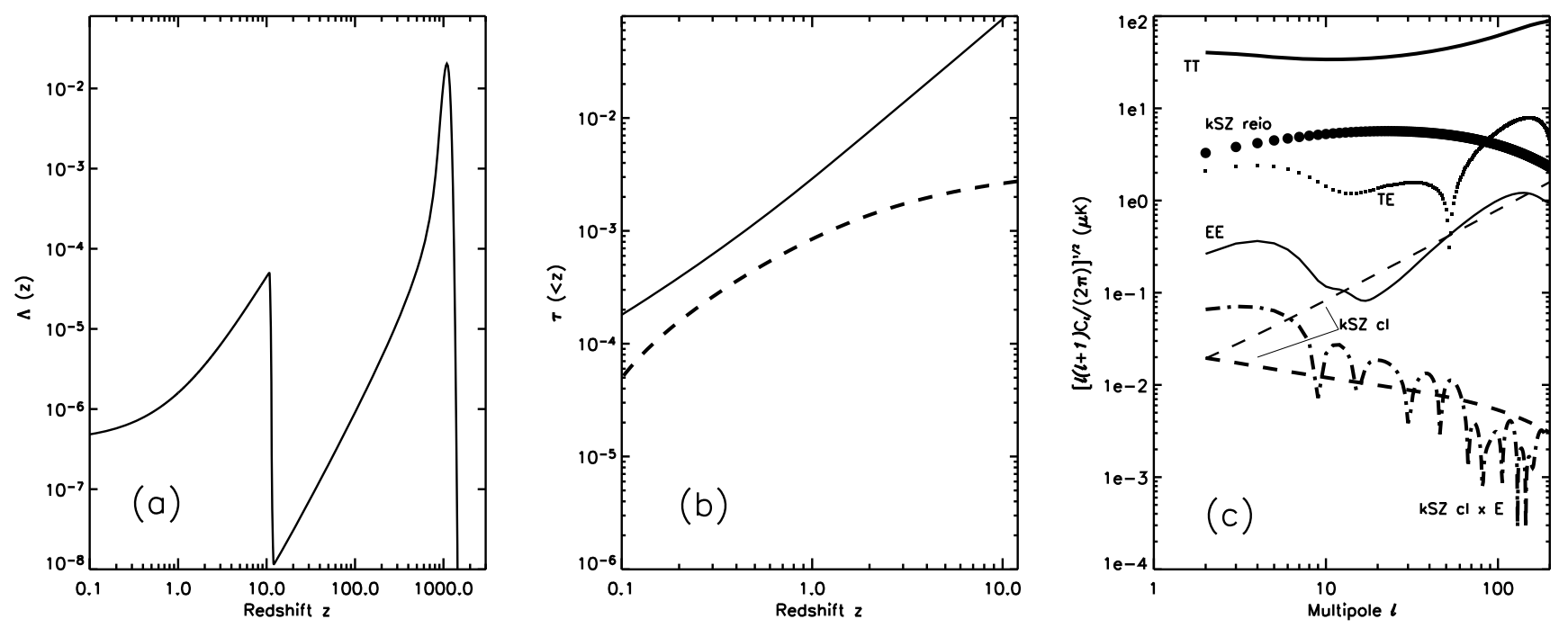

Fig. 1. (Left panel) a) Visibility function versus redshift under WMAP5 cosmogony. (Middle panel) b) Thomson optical depth versus redshift due to all electrons (solid line) and to those electrons contained in halos more massive than $5 \times 10^{12} M_{\odot}$ (dashed line). (Right panel) c) Showing square root of angular power spectra, note the $\mu K$ units. Thick solid line: total CMB temperature anisotropy amplitude. Filled circles: total kSZ induced temperature fluctuations generated during and after reionization. Thin solid line: total E polarization mode power spectrum. Thick dashed line: correlation term of the Thomson scattering induced fluctuations generated in galaxy clusters. Thin dashed line: Poisson term of the Thomson scattering induced fluctuations in galaxy clusters. Dot-dashed line: cross-correlation between the total E-mode polarization (thin solid line) and the correlated part of the kSZ generated in galaxy groups and clusters (thick dashed line).

temperature fluctuations do not depend on the frequency, and therefore they cannot be easily distinguished from the intrinsic CMB temperature fluctuations. However, the kSZ further modifies the CMB photon field by introducing additional polarization, and this should provide another way to isolate this effect.

In this work we exploit the anisotropic nature of the Thomson scattering (for which linear polarization is introduced in the CMB), and use it as a tool to reveal the bulk flows of the missing baryons in the low redshift universe, $(z<2-3)$. For that, we need to assume that the peculiar motion of clusters and groups are correlated to the velocities of the surrounding matter (just as the linear theory predicts), and use future measurements on halos as a kSZ template for all baryons. In Sect. 2 we compute the kSZ and the polarization generated during and after reionization, and the cross-correlation between both. In Sect. 3 we compute the $\mathrm{kSZ}$ produced by the halo population and the signal to noise $(S / N)$ ratio for its cross-correlation to the allsky E-mode polarization. We discuss our results and conclude in Sect. 4. Throughout this paper, we use a cosmological parameter set corresponding to the WMAP5 $+\Lambda$ CDM model (Hinshaw et al. 2008).

\section{The large scale secondary anisotropies after reionization}

According to WMAP data, the first stars must have reionized the IGM at around $z_{\text {reio }} \simeq 11$ (Hinshaw et al. 2008). Since then, CMB photons and electrons have interacted again via Thomson scattering. As during recombination, this scattering preserves the black body spectrum of the $\mathrm{CMB}$, but changes the direction of the CMB photons, and, in the presence of a non-vanishing quadrupole of the CMB intensity, in also induces linear polarization. This process is the cause of a partial blurring of the anisotropies generated at the surface of last scattering and also the generation of new anisotropies at scales comparable to the horizon at $z=z_{\text {reio }}\left(\theta \sim 20^{\circ}\right.$ or $l \sim 10$, Siuniaev 1977). (One should also consider that, if reionization proceeded first in those most overdense regions hosting the first stars, then it must also have left a patchy signature on the CMB small scale anisotropies. However, in this work we shall neglect these small angle fluctuations). The temperature anisotropies introduced in the CMB intensity were caused by the peculiar motions of the scatterers (electrons) with respect to the CMB, (i.e., the kSZ effect). A simple expression for these temperature anisotropies is given by the following integral of the projection of the peculiar velocity along the line of sight $\hat{\boldsymbol{n}}$ :

$\frac{\delta T}{T_{0}}(\hat{\boldsymbol{n}}) \simeq \int_{\eta_{\text {reio }}}^{\eta_{0}} \mathrm{~d} \eta \Lambda(\eta)\left(-\frac{\hat{\boldsymbol{n}} \cdot \boldsymbol{v}_{\mathrm{e}}(\hat{\boldsymbol{n}}, \eta)}{c}\right)$,

(note that we are neglecting the few percent contribution of the polarization terms). As in Seljak \& Zaldarriaga (1996), in this equation $\eta$ is the conformal time (related to the coordinate time via $\mathrm{d} \eta=\mathrm{d} t / a(t)$, with $a$ the cosmological scale factor), and $\eta_{\text {reio }}, \eta_{0}$ are its values at reionization and present, respectively. Dots will denote derivatives with respect to the conformal time. The (proper) electron peculiar velocity is given by $\boldsymbol{v}_{\mathrm{e}}(\eta)$, and its Fourier counterpart can be related to the underlying linear density perturbation field by means of the continuity equation. These peculiar velocities describe the large scale bulk flows of matter that are generated by the linear perturbations in the matter density field. The visibility function is defined as $\Lambda(\eta)=\dot{\tau}(\eta) \exp (-\tau(\eta))$, with $\tau(\eta)$ the Thomson optical depth given by $\tau(\eta) \equiv \int_{\eta}^{\eta_{0}} \mathrm{~d} \eta^{\prime} a\left(\eta^{\prime}\right) \sigma_{\mathrm{T}} n_{\mathrm{e}}\left(\eta^{\prime}\right)$. In this expression, $n_{\mathrm{e}}(\eta)$ is the background electron number density and $\sigma_{\mathrm{T}}$ the Thomson cross section. The Thomson opacity is given by $\dot{\tau}(\eta) \equiv a(\eta) \sigma_{T} n_{\mathrm{e}}(\eta)$. The visibility function $\Lambda(\eta)$ denotes the probability that a CMB photon was last scattered at the epoch given by $\eta$. In panel (a) of Fig. 1 it can be seen that the visibility function peaks at recombination redshift $z \simeq 1100$ (as first predicted by Sunyaev \& Zeldovich 1970), although it has a second 
peak at $z=z_{\text {reio }} \simeq 10$, from the new Thomson scattering taking place after the stars reionize the IGM (Siuniaev 1977). Note that the optical depth behaves differently, increasing steadily up to the reionization redshift (solid line of panel (b) in Fig. 1).

The anisotropic nature of the Thomson scattering introduces linear polarization as long as the CMB intensity has a nonvanishing local quadrupole. In CMB studies, it is customary to map the standard $Q$ and $U$ polarization Stokes parameters on the (rotation invariant) $E$ and $B$ modes (Zaldarriaga \& Seljak 1997; Kamionkowski et al. 1997), which behave with opposite parity properties versus spatial reflections. Pure Thomson scattering should produce no $B$ mode polarization, and hereafter this mode will be neglected. Since the evolution of the intensity and E-mode polarization anisotropies are coupled by the sum of the intensity quadrupole and the polarization monopole and quadrupole, both quantities will be correlated, and this crosscorrelation has already been measured by WMAP, (Hinshaw et al. 2008).

Using a modified version of a standard Boltzmann code (like, e.g., CMBFAST Seljak \& Zaldarriaga 1996) it is straightforward to compute the temperature and E-mode polarization anisotropies generated during and after reionization, and the cross-correlation between them. The results, computed under WMAP5 cosmology (Hinshaw et al. 2008), are displayed in Fig. 1c. The total intensity (TT) and E polarization (EE) angular power spectra are displayed by the thick and thin solid lines, respectively, whereas the cross-correlation between the intensity and E polarization is given by the dotted line (TE). The temperature anisotropies induced by the $\mathrm{kSZ}$ during and after reionization are given by the filled circles, and the generation of polarization at the same cosmic epochs is the responsible for the low $l(l \sim 5-8)$ bump in the EE spectrum. Although the WMAP ${ }^{1}$ mission has barely measured it, the forthcoming Planck ${ }^{2}$ satellite should be able to provide an accurate estimate of the EE angular power spectrum (and this shall be our assumption hereafter).

\section{The clusters as probes of bulk flows}

As mentioned in the Introduction, there are two major aspects of our understanding of the local Universe that still are pending confirmation: one is the detection of the eight ninths of baryons that have not yet been found (Fukugita \& Peebles 2004), the another is the measurement of the cosmological bulk flows. With the advent of high resolution and high sensitivity CMB experiments like ACT (Fowler \& ACT Collaboration 2006), SPT (Ruhl et al. 2004) or APEX (Dobbs et al. 2006), bulk flows may be detected by looking at the kSZ in galaxy clusters. These structures contain large reservoirs of gas, which constitute sources for relatively large values of optical depth, $\left(\tau \sim 10^{-4}, 10^{-3}\right)$. Nevertheless, one must have in mind that the kSZ effect is not the only physical mechanism to be found in clusters. Indeed, given the fact that, in these halos, gas can reach temperatures at the level of a few $\mathrm{KeV}$, inverse Compton scattering in which hot electrons transfer energy to CMB photons (tSZ) becomes of relevance as well. However, the brightness temperature fluctuations introduced by this effect cross from negative to positive values at a frequency close to $218 \mathrm{GHz}$. For this reason, this frequency becomes an ideal window for searching for the $\mathrm{kSZ}$ in galaxy clusters.

\footnotetext{
1 WMAP URL site: http: //map.gsfc.nasa.gov

2 Planck's URL site:

http://www .rssd.esa . int/index . php?project=planck
}

The kSZ effect generated in the galaxy cluster population has been studied extensively (Peel 2006; Hernández-Monteagudo et al. 2006a; Bhattacharya \& Kosowsky 2007, 2008, etc.). In particular, in Hernández-Monteagudo et al. (2006a) an analysis of the correlation properties of the cluster peculiar motion was provided. They found that the typical correlation length of these peculiar velocities (i.e., the typical size of a bulk flow) is typically $20 \mathrm{~h}^{-1} \mathrm{Mpc}$ in comoving units. This means that a large fraction of the gas that is surrounding the clusters is actually comoving with it. This can be envisioned as flows of matter (containing clusters, groups and diffuse gas) falling into the (future) supercluster's potential well, and generating a kSZ dipole pattern at its center as different flows merge in that region (Diaferio et al. 2000). This is related to the basic assumption of this work: peculiar motion of baryons in a given region is well described by the peculiar motion of the largest galaxy groups and clusters within the same region. When computing the all sky kSZ angular power spectrum generated by clusters, Hernández-Monteagudo et al. (2006a) found that although the this could be decomposed in four terms, only two of them give rise to most of the power: a correlation term (generated by the bulk flows) was dominant at the very large angular scales, whereas the anisotropy induced by the Poisson statistics would dominate at smaller scales. Both correlation and Poisson terms are shown by the thick and thin dashed lines in Fig. 1c. The former has been computed using a modified version of the CMBFAST code where the Thomson opacity generated in clusters at a given $\eta$ reads (Hernández-Monteagudo et al. 2006a):

$$
\dot{\tau}=a(\eta) \sigma_{T} \int_{M \min }^{M_{\max }} \mathrm{d} M \frac{\mathrm{d} n}{\mathrm{~d} M} N_{\mathrm{e}}(M) b_{v}
$$

The symbol $N_{\mathrm{e}}(M)=M /\left(m_{\mathrm{p}} f_{Y}\right) \Omega_{\mathrm{b}} / \Omega_{\mathrm{m}}$ denotes the number of electrons in a halo of mass $M$, with $m_{\mathrm{p}}$ the proton mass, $f_{Y}=1-Y / 2$ the helium fraction correction factor and $\Omega_{\mathrm{b}}$, $\Omega_{\mathrm{m}}$ the baryon and matter density parameters, respectively. The halo mass function $\mathrm{d} n / \mathrm{d} M$ is approximated by that of Sheth \& Tormen (1999). Motivated by Peel (2006), the velocity bias was approximated by $b_{v}=1.3$ for all halos (although the particular choice of this parameter has very little impact on our results, as we shall see below). We set $M_{\max }=10^{16} M_{\odot}$, and compared our results for different choices of the minimum mass $M$ min. In the middle panel of Fig. 1 the dashed line shows the optical depth generated by halos more massive than $5 \times 10^{12} M_{\odot}$ : most of the contribution $(\sim 60 \%)$ comes from $z<3$, and the contribution from (the more massive) galaxy groups and clusters is shifted even further into the low $z$ range. On the other hand, the Poisson term can easily be found to be

$$
\begin{aligned}
C_{l}^{k S Z, \xi}= & \int_{\eta_{\text {reio }}}^{\eta\left(z_{\min }\right)} \mathrm{d} \eta \eta^{2} \int_{M \min }^{M_{\max }} \mathrm{d} M \\
& \times \frac{\mathrm{d} n}{\mathrm{~d} M}\left(\frac{\sigma_{\mathrm{T}} N_{\mathrm{e}}(M) b_{v} \sigma_{v}(M, \eta)}{d_{\text {Ang }}^{2}(\eta)}\right)^{2}\left(1+\frac{1}{\xi^{2}}\right),
\end{aligned}
$$

where $d_{\text {Ang }}(\eta)$ denotes angular diameter distance and $\sigma_{v}(M, \eta)$ is the time dependent rms line of sight peculiar velocity of an object of mass $M$,

$\sigma_{v}^{2}(M, \eta)=\frac{1}{3}\left(H(\eta)\left|\frac{\mathrm{d} \mathcal{D}_{\delta}}{\mathrm{d} z}\right|\right)^{2} \int \mathrm{d} k k^{2} \frac{P_{\mathrm{m}}(k)}{2 \pi^{2} k^{2}}|W(k R(M))|^{2}$

The present linear matter power spectrum is given by $P_{\mathrm{m}}(k)$, the linear density growth factor by $\mathcal{D}_{\delta}$, the Hubble parameter by $H(\eta)$, and the Fourier window function of a top hat filter corresponding to an object of mass $M$ by $W(k R(M)$ ) (see 

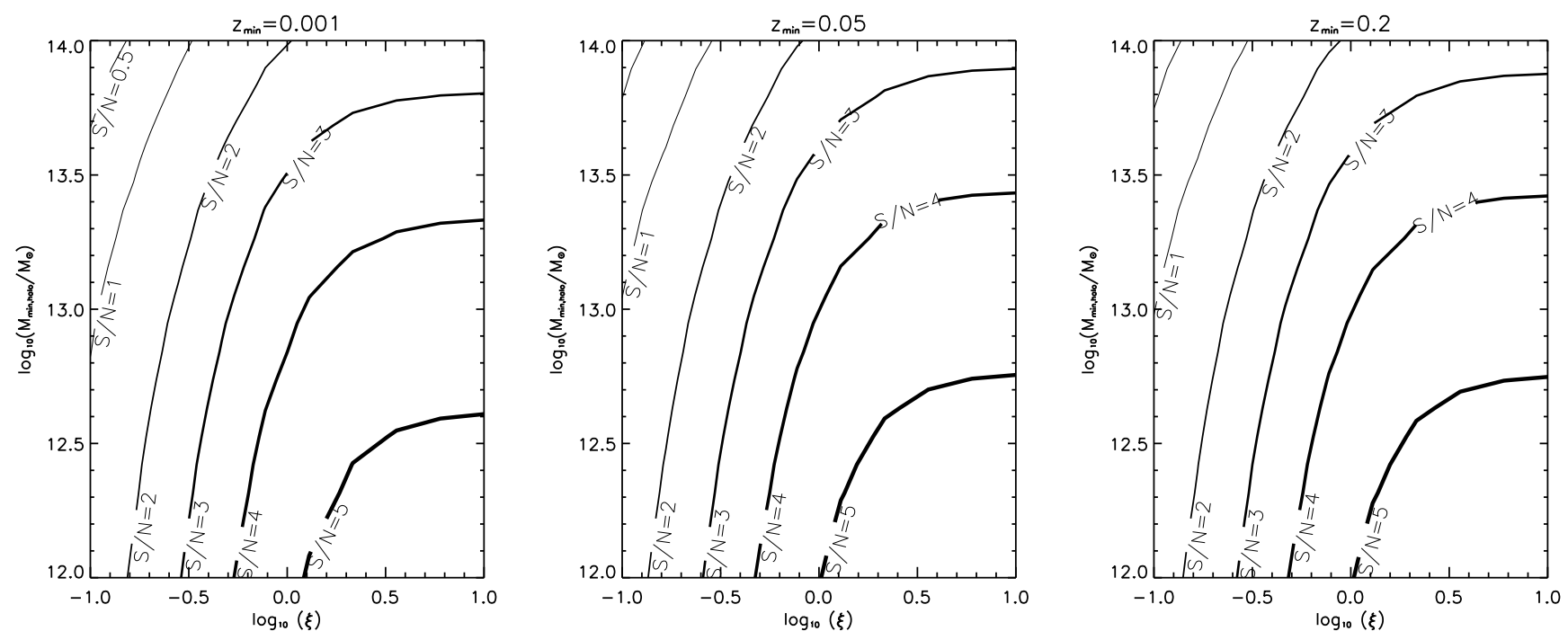

Fig. 2. The total $S / N$ ratio for the correlation of the E-mode polarization with the low redshift $(z<3-4)$ kSZ generated by bulk flows in galaxy groups and clusters. Horizontal axes display the decimal logarithm of the individual group/cluster kSZ measurement $S / N$ ratio $(\xi)$, whereas vertical axes display the decimal logarithm of the minimum mass of groups/clusters considered. Due to Poisson/shot noise, a low choice for $z_{\min }$ makes the high total $S / N$ regions shrink, and this also happens if $z_{\min }$ is chosen too large and too much information is unused. The optimal minimum redshift is around $z_{\min } \simeq 0.05$, and the maximum $S / N$ ratio achievable for perfect $\mathrm{E}$ and $\mathrm{kSZ}$ surveys is $\sim 5.8$. Note that we are assuming $f_{\text {sky }}=1$.

Hernández-Monteagudo et al. 2006a, for details). Note that in Eq. (3), $\xi$ denotes the signal to noise ratio of the $\mathrm{kSZ}$ detection in a single cluster/group. As noise we regard any signal present at the cluster position that is not due to the correlated component of the kSZ (e.g., kSZ caused by thermal velocities, $\mathrm{tSZ}$ residuals, intrinsic $\mathrm{CMB}$, radio source emission, IR source emission, instrumental noise, etc). Note as well the low redshift limit given by $z_{\min }$ in Eq. (3). Therefore, this equation accounts for both the Poissonian statistics and the error in the kSZ recovery at cluster positions. Only in the case $\xi \rightarrow \infty$ we recover the pure Poissonian term.

We are interested in the correlated component of the cluster kSZ, i.e., in the velocity component that should be comoving with the surrounding matter. If this is indeed the case, then the pattern of the kSZ in clusters must be very similar to that of the kSZ generated by all electrons (filled circles in Fig. 1), and therefore it must be closely correlated to the $E$ mode of the polarization anisotropies generated during and after reionization (i.e., the low $l$ bump). The actual amplitude of this cross-correlation will depend on the redshift range that a given set of halos is probing, and the signal to noise $(S / N)$ ratio of this cross-correlation for a particular multipole $l$ is provided by

$$
\left(\frac{S}{N}\right)_{l}^{2}=\frac{(2 l+1) f_{\mathrm{sky}}\left(C_{l}^{k S Z, c l-E}\right)^{2}}{C_{l}^{E E}\left(C_{l}^{k S Z, c l}+C_{l}^{k S Z, \xi}\right)+\left(C_{l}^{k S Z, c l-E}\right)^{2}} .
$$

The symbol $f_{\text {sky }}$ refers to the fraction of the sky covered by an experiment, and $C_{l}^{k S Z, c l}$ and $C_{l}^{k S Z, \xi}$ denote, respectively, the angular power spectrum of the correlated part and the noise of the $\mathrm{kSZ}$ generated in galaxy clusters. The cross-correlation between the kSZ in halos and the E-mode is given by $C_{l}^{k S Z, c l-E}$. If $f_{\text {sky }}=1$, the total $S / N$ is given by the sum of the squares of these quantities, i.e., $S / N=\left[\sum_{l}(S / N)_{l}^{2}\right]^{1 / 2}$. It turns out that the actual amplitude of this $S / N$ ratio is strongly dependent on the relative amplitude of the Poisson term with respect to the correlation term (and this makes the $S / N$ independent of parameters like the velocity bias $b_{v}$ ). Due to the $\eta^{2} / d_{\text {Ang }}^{4} \propto 1 / \eta^{2}$ dependence in
Eq. (3), the Poisson term is less important for higher values of the minimum redshift considered $z_{\min }$. Further, it depends linearly on the number density of objects, as opposed to the correlation term, which depends on the number density squared. Therefore, for lower values of the threshold mass $M_{\min }$ the relative weight of the Poisson term versus the correlation one should diminish. This is shown in Fig. 2. Since this correlation arises around halos at low and moderate redshifts, the matter behind this signal is different from that generating the anti crosscorrelation between CMB anisotropies and HI $21 \mathrm{~cm}$ observations.

\section{Discussion and conclusions}

The horizontal axis of Fig. 2 corresponds to the $S / N$ ratio at which the $\mathrm{kSZ}$ can be determined in a single halo, i.e., $\xi$ in Eq. (3). The number of potential contaminants when measuring the kSZ in a halo is relatively high: the tSZ has a definite spectral dependence, and should be close to zero at $v=$ $218 \mathrm{GHz}$, however asymmetries in the frequency response of the experiment together with relativistic effects (Itoh \& Nozawa 2004) will inevitably leave some residuals. However, as shown in Hernández-Monteagudo et al. (2006a), its weight relative to the kSZ decreases for the most numerous low mass halos, for which the relativistic tSZ corrections are also less important. Internal motion within the halos (which, as found by Diaferio et al. 2005, is only relevant for slow halos) should average out when integrating over the total halo solid angle. The uncorrelated component of the kSZ could be significant in those systems where the velocity of halos are thermalized; however this almost never happens for the galaxy groups and clusters being targeted here, since superclusters are structures that are not yet relaxed. Nevertheless, Peel (2006) found that, in his cosmological simulations, the peculiar velocities of haloes showed correlation properties that differed substantially from linear theory predictions, even at high redshift. If this is indeed the case, then the issue would be whether the baryons surrounding halos would have their peculiar velocities affected in the same way or 
not. We shall address this issue in future work. The impact of IR and radio sources is a priori significant, provided that these sources are correlated to the halos where the $\mathrm{kSZ}$ is produced. However, multifrequency observations should provide a good insight into their spectral indexes, and therefore their contribution in the $218 \mathrm{GHz}$ channel should be characterized down to a given accuracy level. How this compares to the actual kSZ signal is something to be reliably evaluated only once real data becomes available. The intrinsic CMB generated at the last scattering surface is another potential source of contamination. As shown in Fig. 6 of Hernández-Monteagudo et al. (2006a), its residuals remain in the few $\mu \mathrm{K}$ level if the cluster/group size is not larger than 2-3 arcmins, which should be the case for most of clusters and groups in the sky. Therefore this source of contamination is likely to be less relevant than the rest.

The importance of each of these contaminants will determine which range of the $\xi$ horizontal axis in Fig. 2 one should look at. Due to the largest amplitude of Poissonian fluctuations at low values of $z_{\mathrm{min}}$, it is convenient not to consider too nearby clusters and groups. From this figure it is possible to find at which values of $\xi$ the $S / N$ ratio starts to be dominated by Cosmic Variance (the horizontal plateau of the contour levels at high $\xi$ ). Likewise, the steep vertical descent of these contours at low $\xi$ reflects the limit where $S / N$ is exclusively limited by the accuracy of the $\mathrm{kSZ}$ measurements. The requirements for a convincing detection of the signature of the local baryon bulk flows (say $S / N>3$ ) involve either a large value of $\xi$ or a low value of $M_{\min }$, but nevertheless these are not unrealistic. For instance, the Planck satellite should provide a cleaned CMB map in a very large fraction of the sky, and with it, estimations of the $\mathrm{kSZ}$ at the cluster and group positions. These estimates should mostly be limited by the beam size and the un-subtracted point source emission. By looking at the Compton distortion $y$ map (obtained from multifrequency observations of the tSZ), one should be able to find the position of not only the largest clusters, but also of those objects that lie closer to the confusion limit, and whose signal can be picked up statistically via the cross-correlation. If this $y$-map provides the position and the cleaned CMB map the $\mathrm{kSZ}$ estimates (of at least $\xi \sim 1$ ) for all clusters more massive than $10^{14} M_{\odot}$, then Planck alone would already yield a total $S / N$ between 2 and 3. Likewise, upcoming Dark Energy surveys like Pan-STARRS ${ }^{3}, \mathrm{DES}^{4}$ or PAU-BAO ${ }^{5}$, or X-ray surveys like eROSITA $^{6}$ should probe the relevant redshift range, and independently provide catalogs of group and cluster candidates. At the same time, high resolution CMB experiments like ACT, SPT or APEX would contribute more accurate kSZ estimates. Furthermore, future B-mode polarization experiments should cover large fractions of the sky with very high sensitivity, and provide better understanding of the E-mode of the CMB polarization. The maximum achievable value of the total $S / N$ is relatively small (around 5.8), and therefore a large sky coverage is required, since most of the signal arises at $l<10$. Our computations are within the regime of linear theory. Corrections due to patchy reionization are non linear and therefore should appear at much smaller angular scales. They should also arise at redshifts higher than those corresponding to the kSZ addressed in this work, $(z<3-4)$. We must also stress again that a cosmic variance limited EE power spectrum has been assumed, at least in those same large angular scales. Very soon, the Planck mission should be able to tell whether this is a sensible assumption or not.

Acknowledgements. C.H.M. acknowledges useful conversations with D.N. Spergel.

\section{References}

Atrio-Barandela, F., Kashlinsky, A., \& Mücket, J. P. 2004, ApJ, 601, L111 Atrio-Barandela, F., Mücket, J. P., \& Génova-Santos, R. 2008, ApJ, 674, L61 Battistelli, E. S., et al. 2006, [arXiv:astro-ph/0603702]

Benson, B. A., Church, S. E., Ade, P. A. R., et al. 2003, ApJ, 592, 674

Bhattacharya, S., \& Kosowsky, A. 2007, ApJ, 659, L83

Bhattacharya, S., \& Kosowsky, A. 2008, Phys. Rev. D, 77, 083004

Böhringer, H., Voges, W., Huchra, J. P., et al. 2000, ApJS, 129, 435

Cen, R., \& Ostriker, J. P. 1999, ApJ, 514, 1

Cen, R., \& Ostriker, J. P. 2005, [arXiv: astro-ph/0601008]

Croft, R. A. C., Weinberg, D. H., Katz, N., \& Hernquist, L. 1998, ApJ, 495, 44

Diaferio, A., Sunyaev, R. A., \& Nusser, A. 2000, ApJ, 533, L71

Diaferio, A., Borgani, S., Moscardini, L., et al. 2005, MNRAS, 356, 1477

Dobbs, M., Halverson, N. W., Ade, P. A. R., et al. 2006, New Astron. Rev., 50, 960

Fowler, J. W., \& ACT Collaboration 2006, BAAS, 38, 1227

Fukugita, M., \& Peebles, P. J. E. 2004, ApJ, 616, 643

Génova-Santos, R., Rubin o-Marti'n, J. A., Rebolo, R., et al. 2005, MNRAS, 363,79

Genova-Santos, R., et al. 2008, [arXiv:0804.0199]

Hernández-Monteagudo, C., Verde, L., Jimenez, R., \& Spergel, D. N. 2006a, ApJ, 643, 598

Hernández-Monteagudo, C., Trac, H., Verde, L., \& Jimenez, R. 2006b, ApJ, 652, L1

Hinshaw, G., et al. 2008, [arXiv: 0803.0732]

Itoh, N., \& Nozawa, S. 2004, A\&A, 417, 827

Kamionkowski, M., Kosowsky, A., \& Stebbins, A. 1997, Phys. Rev. D, 55, 7368

Kashlinsky, A., \& Atrio-Barandela, F. 2000, ApJ, 536, L67

Kull, A., \& Böhringer, H. 1999, A\&A, 341, 23

McDonald, P., Seljak, U., Cen, R., et al. 2005, ApJ, 635, 761

Nicastro, F., Mathur, S., Elvis, M., et al. 2005a, ApJ, 629, 700

Nicastro, F., Mathur, S., Elvis, M., et al. 2005b, Nature, 433, 495

Peel, A. C. 2006, MNRAS, 365, 1191

Ruhl, J., Ruhl, J., Ade, P. A. R., Carlstrom, J. E., et al. 2004, SPIE, 5498, 11

Seljak, U., \& Zaldarriaga, M. 1996, ApJ, 469, 437

Sheth, R. K., \& Tormen, G. 1999, MNRAS, 308, 119

Siuniaev, R. A. 1977, Sov. Astron. Lett., 3, 268

Sunyaev, R. A., \& Zeldovich, Y. B. 1970, Ap\&SS, 7, 3

Sunyaev, R. A., \& Zeldovich, Y. B. 1972, Comm. Astrophys. Space Phys., 4, 173

Sunyaev, R. A., \& Zeldovich, I. B. 1980, MNRAS, 190, 413

Zaldarriaga, M., \& Seljak, U. 1997, Phys. Rev. D, 55, 1830

Zappacosta, L., Mannucci, F., Maiolino, R., et al. 2002, A\&A, 394, 7

\footnotetext{
3 Pan-STARRS' URL site: http://pan-starrs.ifa.hawaii. edu/public/

${ }^{4}$ DES's URL site: http://www . darkenergysurvey .org/

5 PAU-BAO's URL site: http://wwW.ice.csic.es/research/ PAU/

${ }^{6}$ eROSITA's URL site: http://www.mpe.mpg.de/projects. html\#erosita
} 